\title{
Article \\ Continuity of Cancer Care: The Surgical Experience of Two Large Cancer Hubs in London and Milan
}

\author{
Maria J. Monroy-Iglesias ${ }^{1, *,+}{ }^{\dagger}$, Marta Tagliabue ${ }^{2,3,+}{ }^{\oplus}$, Harvey Dickinson ${ }^{4}$, Graham Roberts ${ }^{4}$, \\ Rita De Berardinis ${ }^{2, *,+}\left(\mathbb{D}\right.$, Beth Russell ${ }^{1} \mathbb{1}$, Charlotte Moss ${ }^{1}$, Sophie Irwin ${ }^{4}$, Jonathon Olsburgh ${ }^{5}$, \\ Ivana Maria Francesca Cocco ${ }^{6}$, Alexis Schizas ${ }^{6}$, Sarah McCrindle ${ }^{7}$, Rahul Nath ${ }^{8}$, Aina Brunet ${ }^{9}$, Ricard Simo ${ }^{9}$, \\ Chrysostomos Tornari ${ }^{9}$ (D) Parthi Srinivasan ${ }^{10}$, Andreas Prachalias ${ }^{10}$, Andrew Davies ${ }^{4}$, Jenny Geh ${ }^{11}$, \\ Stephanie Fraser ${ }^{12}$, Tom Routledge ${ }^{12}$, RuJun Ma ${ }^{12}$, Ella Doerge ${ }^{13}$, Ben Challacombe ${ }^{13}$, Raj Nair ${ }^{13}$, \\ Marios Hadjipavlou ${ }^{13}$, Rosaria Scarpinata ${ }^{14}$, Paolo Sorelli ${ }^{15}$, Saoirse Dolly ${ }^{7} \mathbb{D}$, Francesco Alessandro Mistretta ${ }^{16}{ }^{(D)}$, \\ Gennaro Musi ${ }^{16,17}$, Monica Casiraghi ${ }^{18}{ }^{1}$, Alessia Aloisi ${ }^{19}$, Andrea Dell'Acqua ${ }^{19}$, Donatella Scaglione ${ }^{20}$, \\ Stefania Zanoni ${ }^{20}$, Daniele Rampazio Da Silva ${ }^{20}$, Daniela Brambilla ${ }^{20}$, Raffaella Bertolotti ${ }^{20}$, Giulia Peruzzotti ${ }^{20}$, \\ Angelo Maggioni ${ }^{19}$, Ottavio de Cobelli 16,17, Lorenzo Spaggiari ${ }^{17,18}$, Mohssen Ansarin ${ }^{2}$, Fabrizio Mastrilli ${ }^{21}$, \\ Sara Gandini ${ }^{22}$ (D) Urvashi Jain ${ }^{23}$, Hisham Hamed ${ }^{23}$, Kate Haire ${ }^{4}$ and Mieke Van Hemelrijck ${ }^{1}$
}

Citation: Monroy-Iglesias, M.J.; Tagliabue, M.; Dickinson, H.; Roberts, G.; De Berardinis, R.; Russell, B.; Moss, C.; Irwin, S.; Olsburgh, J.; Cocco, I.M.F.; et al. Continuity of Cancer Care: The Surgical Experience of Two Large Cancer Hubs in London and Milan. Cancers 2021, 13, 1597. https://doi.org/10.3390/cancers 13071597

Academic Editors: Brendon Coventry and David Wong

Received: 26 February 2021

Accepted: 26 March 2021

Published: 30 March 2021

Publisher's Note: MDPI stays neutral with regard to jurisdictional claims in published maps and institutional affiliations.

Copyright: (C) 2021 by the authors Licensee MDPI, Basel, Switzerland. This article is an open access article distributed under the terms and conditions of the Creative Commons Attribution (CC BY) license (https:/ / creativecommons.org/licenses/by/ $4.0 /)$.
1 Faculty of Life Sciences and Medicine, Translational Oncology \& Urology Research (TOUR), King's College London, London WC2R 2LS, UK; beth.russell@kcl.ac.uk (B.R.); charlotte.moss@kcl.ac.uk (C.M.); mieke.vanhemelrijck@kcl.ac.uk (M.V.H.)

2 Division of Otolaryngology and Head and Neck Surgery, European Institute of Oncology IRCCS, 20122 Milan, Italy; marta.tagliabue@ieo.it (M.T.); Mohssen.Ansarin@ieo.it (M.A.)

3 Department of Biomedical Sciences, University of Sassari, 07100 Sassari, Italy

4 South East London Cancer Alliance, London SE1 9RT, UK; harvey.dickinson@gstt.nhs.uk (H.D.); graham.roberts@gstt.nhs.uk (G.R.); sophie.irwin@gstt.nhs.uk (S.I.); Andrew.Davies@gstt.nhs.uk (A.D.); Kate.Haire@gstt.nhs.uk (K.H.)

5 Department of Nephrology and Transplantation, Guy's \& St Thomas' NHS Foundation Trust, London SE1 9RT, UK; Jonathon.Olsburgh@gstt.nhs.uk

6 Department of Colorectal Surgery, Guy's and St Thomas' NHS Foundation Trust, London SE1 9RT, UK; Francesca.Cocco@gstt.nhs.uk (I.M.F.C.); Alexis.Schizas@gstt.nhs.uk (A.S.)

7 Department of Medical Oncology, Guy's and St Thomas' NHS Foundation Trust, London SE1 9RT, UK; Sarah.Mccrindle@gstt.nhs.uk (S.M.); Saoirse.Dolly@gstt.nhs.uk (S.D.)

8 Department of Gynaecological Oncology, Guy's and St Thomas' NHS Foundation Trust, London SE1 9RT, UK; Rahul.Nath@gstt.nhs.uk

9 Department of Otorhinolaryngology Head and Neck Surgery, Guy's and St Thomas' NHS Foundation Trust, London SE1 9RT, UK; Aina.Brunet@gstt.nhs.uk (A.B.); Ricard.Simo@gstt.nhs.uk (R.S.); Chrysostomos.Tornari@gstt.nhs.uk (C.T.)

10 Department of Liver Studies, King's College Hospital, London SE5 9RS, UK; Parthi.Srinivasan@nhs.net (P.S.); Andreas.Prachalias@nhs.net (A.P.)

11 Department of Plastic Surgery, Guy's and St Thomas' NHS Foundation Trust, London SE1 9RT, UK; Jenny.Geh@gstt.nhs.uk

12 Department of Thoracic Surgery, Guy's and St Thomas' NHS Foundation Trust, London SE1 9RT, UK; Stephanie.Fraser@gstt.nhs.uk (S.F.); Tom.Routledge@gstt.nhs.uk (T.R.); Rujun.Ma@gstt.nhs.uk (R.M.)

13 Department of Urology, Guy's and St Thomas' NHS Foundation Trust, London SE1 9RT, UK; Ella.Doerge@gstt.nhs.uk (E.D.); Ben.Challacombe@gstt.nhs.uk (B.C.); Raj.Nair@gstt.nhs.uk (R.N.); Marios.Hadjipavlou@gstt.nhs.uk (M.H.)

14 Department of Colorectal Surgery, King's College Hospital, London SE5 9RS, UK; Rosaria.Scarpinata@nhs.net

15 Department of Colorectal Surgery, Lewisham and Greenwich NHS Trust, London SE13 6LH, UK; Paolo.Sorelli@nhs.net

16 Division of Urology, European Institute of Oncology IRCCS, 20122 Milan, Italy; FrancescoAlessandro.Mistretta@ieo.it (F.A.M.); Gennaro.Musi@ieo.it (G.M.); Ottavio.deCobelli@ieo.it (O.d.C.)

17 Department of Oncology and Hemato-Oncology, University of Milan, 20122 Milan, Italy; Lorenzo.Spaggiari@ieo.it

18 Division of Thoracic Surgery, European Institute of Oncology IRCCS, 20122 Milan, Italy; Monica.Casiraghi@ieo.it

19 Division of Gynaecological Surgery, European Institute of Oncology IRCCS, 20122 Milan, Italy; Alessia.aloisi@ieo.it (A.A.); Andrea.DellAcqua@ieo.it (A.D.); Angelo.Maggioni@ieo.it (A.M.)

20 Division of Data Management, European Institute of Oncology IRCCS, 20122 Milan, Italy; Donatella.Scaglione@ieo.it (D.S.); Stefania.Zanoni@ieo.it (S.Z.); daniele.rampaziodasilva@ieo.it (D.R.D.S.); Daniela.Brambilla@ieo.it (D.B.); Raffaella.Bertolotti@ieo.it (R.B.); Giulia.Peruzzotti@ieo.it (G.P.) 
21 Medical Administration, European Institute of Oncology, IRCCS, 20122 Milan, Italy; Fabrizio.Mastrilli@ieo.it

22 Department of Experimental Oncology, European Institute of Oncology IRCCS, 20122 Milan, Italy; Sara.Gandini@ieo.it

23 Department of Breast Surgery, Guy's and St Thomas' NHS Foundation Trust, London SE1 9RT, UK; Jain.urvashi@gstt.nhs.uk (U.J.); Hisham.hamed@gstt.nhs.uk (H.H.)

* Correspondence: maria.j.monroy_iglesias@kcl.ac.uk (M.J.M.-I.); rita.deberardinis@ieo.it (R.D.B.)

+ Maria J. Monroy-Iglesias and Marta Tagliabue contributed equally to this work as co-first authors.

Simple Summary: A better understanding of the reality for cancer patients during the SARS-CoV-2 (COVID-19) pandemic will help us readapt current prediction models. There is a need to dive into rich data sources from apex cancer centres. The aim of our retrospective study was to report on the outcomes of cancer patients receiving radical surgery with curative intent during the first wave of the COVID-19 pandemic. Data from two cancer centres that were at the epicentre of the outbreak from March to September 2020 (as well as a comparator group in 2019) were utilised for this study. We observed that while there was a decline in number of surgeries performed, the implemented COVID-19 minimal pathways were safe for cancer patients requiring surgical treatment.

Abstract: The SARS-CoV-2 (COVID-19) pandemic is having a large effect on the management of cancer patients. This study reports on the approach and outcomes of cancer patients receiving radical surgery with curative intent between March and September 2020 (in comparison to 2019) in the European Institute of Oncology, IRCCS (IEO) in Milan and the South East London Cancer Alliance (SELCA). Both institutions implemented a COVID-19 minimal pathway where patients were required to self-isolate prior to admission and were swabbed for COVID-19 within $72 \mathrm{~h}$ of surgery. Positive patients had surgery deferred until a negative swab. At IEO, radical surgeries declined by $6 \%$ as compared to the same period in 2019 ( $n=1477$ vs. 1560, respectively). Readmissions were required for $3 \%(n=41)$, and $<1 \%(n=9)$ developed COVID-19, of which only one had severe disease and died. At SELCA, radical surgeries declined by $34 \%$ ( $n=1553$ vs. 2336). Readmissions were required for $11 \%(n=36),<1 \%(n=7)$ developed COVID-19, and none died from it. Whilst a decline in number of surgeries was observed in both centres, the implemented COVID-19 minimal pathways have shown to be safe for cancer patients requiring radical treatment, with limited complications and almost no COVID-19 infections.

Keywords: COVID-19; cancer surgery; postoperative outcomes

\section{Introduction}

The new severe acute respiratory syndrome SARS-CoV-2 (COVID-19) was declared a pandemic on 11 March 2020 by the World Health Organisation [1]. This is why healthcare organisations in many countries across the globe have made many significant changes to their healthcare services aiming to face the challenges of the COVID-19 pandemic, leading to a significant disruption in the provision of cancer treatments [2]. The common aim has been to equip institutions to manage the surge of critical patients, and also to minimise face to face contact where possible to reduce further transmission of the virus. Surgical activity in particular has faced many challenges. In the UK and Ireland on 17 March, the National Health Service (NHS) advised to cancel all non-urgent elective surgeries [2]. About two weeks before, in accordance with the anticipated peak in southern Europe, these restrictive measures were also taken in Italy [3,4]. The Italian National Health System (INHS) interrupted all non-urgent surgeries, outpatient consultations, and rehabilitation services [5]. This was initially intended to free up the necessary resources and enable surgical staff to treat COVID-19 patients. Various studies have reported an important decrease in cancer referrals, creating a concern that many cancer patients had a delay in their diagnosis and treatment, resulting in further advancement of their disease [6,7]. Moreover, a recent meta-analysis found that a delay in surgical cancer treatment of four weeks is associated with a $6-8 \%$ increase in the risk of death [8]. Another recent cohort 
study also reported that substantial increases in the number of avoidable cancer deaths in England are to be expected as a result of diagnostic delays due to the COVID-19 pandemic in the UK [9].

The European Institute of Oncology, IRCCS (IEO) in Milan is one of the largest cancer hospitals in Italy. The South East London Cancer Alliance (SELCA) includes three major hospital trusts: Guy's and St. Thomas', Lewisham and Greenwich Trust, and Kings College Hospital. Milan and London were both at the epicentre of the first COVID-19 wave, and their surgical staff were forced to implement strict COVID-19 pathways to continue providing cancer care. However, only few studies have analysed the safety of these pathways.

In the event of another wave of COVID-19, it is important to evaluate the safety of the COVID-19 pathway that was followed for cancer patients requiring radical surgery [10]. Here, we analysed rich data sources from apex Cancer Centres to describe the COVID-19 safety approach and report on the demographic characteristics and surgical outcomes of those cancer patients undergoing radical treatment. This will further inform future clinical guidelines and help readapt current prediction models.

\section{Materials and Methods}

\subsection{Study Population}

At the IEO, the study population consisted of all patients undergoing scheduled radical surgery with curative intent for gynaecological, head and neck (H\&N), thoracic and urological cancers between 1 March and 30 September 2020, as well as the comparable period of 1st March to 30th September 2019. Only patients with complete data were included in the study. In southeast London, all patients undergoing scheduled radical surgery for breast, colorectal, liver, plastic/skin, and upper gastrointestinal cancer, in addition to gynaecological, H\&N, thoracic and urological cancers were included from 23 March to 8 September 2020, and the comparable period in 2019. The periods observed varied due to the earlier start of the COVID-19 pandemic in Italy.

Data collected from all participants included gender, age, socioeconomic status, ethnicity, comorbidities (hypertension, diabetes mellitus (DM), lung conditions, renal impairment, liver conditions, cardiovascular disease (CVD)), performance status (according to the World Health Organisation (WHO)) [11], body mass index (BMI), tumour site, American Society of Anaesthesiologists (ASA) classification [12], surgery time, theatre time, $>24 \mathrm{~h}$ of intensive care unit (ICU) stay, length of stay (LOS), readmissions, complications according to the Clavien Dindo Classification [13], post-operative COVID-19 status, and death by any cause. Data on COVID-19 severity (mild/moderate and severe) and COVID-19-related deaths were only available for the IEO study population. The weekly number of COVID-19 cases in London was extracted from the Public Health England Coronavirus dashboard [14]. The weekly number of COVID-19 cases in Milan was extracted from the Italian Ministry of Health portal $[15,16]$.

\subsection{Patient Pathway-IEO}

Since the initial phases of the pandemic, dedicated personnel were in charge of ascertaining the absence of signs/symptoms of COVID-19 and establishing the urgency/priority of outpatient visits. Hospital entry was only allowed for patients. The use of surgical masks was compulsory, and body temperature was measured by infrared thermometers, where only patients with a body temperature under 37.5 degrees Celsius were allowed to enter. Before admission, all patients underwent a telephone triage to assess their current health status, lack of COVID-19 symptoms (fever, cough, flu-like symptoms, anosmia), and possible contact with COVID-19 positive people or those with symptoms indicative of COVID-19. From April 1st 2020, a nasopharyngeal swab for COVID-19 was collected for all head and neck (HNC) and Thoracic patients at pre-surgical assessment; all the other surgical specialties started to perform nasopharyngeal swab pre-surgical assessment from 1 September 2020. With COVID negative patients, surgery was scheduled within 3 to 5 days 
from the swab. In COVID positive patients, two consecutive negative swabs and 14 days of self-isolation was required to perform surgery.

The anaesthetic protocol was devised to minimise aerosol generation and potential exposure to undetected COVID-19 infection in patients with false negative swab tests. All the involved staff were required to wear full personal protection equipment (PPE) and only the anaesthetist and nurse had access to the operating theatre during the patient's anaesthetic procedure. During the surgery, all staff involved had to remain wearing full PPE throughout the surgical procedure. In the post-operative period, patients were in single rooms with surgical masks and all visiting healthcare professionals were required to use full PPE when entering the room. Updates on the state and outcome of the patients to family members was given via telephone. All patients with COVID-19 suspected symptoms from all specialties were subjected to chest X-ray and/or computed tomography (CT) and nasopharyngeal swab.

\subsection{Patient Pathway-SELCA}

A multidisciplinary team assessed patients' risk profiles according to new government guidance in relation to their co-morbidities and the potential negative effects of COVID-19. If the health risks were deemed too high, patient care was directed to an alternative non-surgical pathway. The need for a post-operative critical care unit (CCU) bed was evaluated, and if deemed too high and prolonged, alternative treatments were considered. An enhanced consenting process was utilised, which included agreed levels of care in the postoperative period with some patients electing not to have CCU care if their condition deteriorated after surgery. Similar to the IEO pathway, all patients were instructed to self-isolate for 14-days to minimise the risk of acquiring COVID-19 infection in the preoperative period. Moreover, two negative swabs were required in surgical pre-assessment in order to proceed to surgery. If the staging computed tomography (CT) scan of the chest identified incidental COVID-19 disease, then surgery would be delayed for 14 days, even if the patients had had two negative swabs.

Prior to the surgery, all patients were intubated in the operating theatre with the anaesthetic team wearing full PPE. Once the endotracheal tube was placed, the surgical team waited $20 \mathrm{~min}$ before entering the theatre, this was to allow for adequate air exchanges to occur and minimise the exposure to aerosol. Throughout the surgery, the team was made up of only consultant surgeons, as junior doctors were deployed to other COVID-19 related duties for the first 2 months of the pandemic. Full PPE was adopted by all theatre staff. Another 20 min were taken after the patient was extubated upon completion of the surgical procedure prior to transfer to the recovery room. Additionally, there was a mandatory simulation training programme for all theatre staff, which included putting on and removing PPE techniques, intubation techniques and failed intubation drills.

Full PPE used by all physicians in both Institutes were filtering face piece 3 (FFP3) mask, in addition to a surgical mask, water-repellent disposable gown or apron, double gloves, and protective goggles or visor. In the surgical theatres, the protocol was to have an area of gowning and de-gowning $[17,18]$. At IEO, also, the health personnel were swabbed every 15 days to detect asymptomatic vectors.

\subsection{Statistical Analyses}

Descriptive statistics were performed to describe baseline socio-demographic and clinical characteristics, surgical, and COVID-19 outcomes. Absolute and relative frequencies for categorical variables, median values, and interquartile (IQR) ranges for continuous variables are reported. Differences in patient characteristics between March to September 2020 and the comparable period in 2019 were evaluated with the Z-score test for two population proportions. 


\section{Results}

\subsection{IEO}

At IEO, there were 1477 radical surgeries with curative intent performed from March to September 2020 (270 for gynaecological, 339 for H\&N, 377 for thoracic, and 491 for urological cancers), compared to 1560 surgeries in the same period in 2019 (274 for gynaecological, 350 for $\mathrm{H} \& \mathrm{~N}, 460$ for thoracic, and 476 urological cancers). There was a decline of $6 \%$ in 2020 compared to 2019. The main decline was seen for thoracic surgery where $18 \%$ less surgeries were performed in 2020 compared to $2019(p=0.01)$. On the other hand, there was a $3 \%$ ( $n=490$ vs. 476$)$ increase in urological cancer surgeries in 2020 compared to $2019(p=0.10)$.

Clinical and demographic characteristics were all comparable between both periods for all cancer types (Tables 1 and A1). Surgical outcomes of IEO patients between 1 March and 30 September 2020 are summarised in Table 2. When looking at ASA grade, $207(14 \%)$ patients had a grade of III or higher (24 (9\%) of gynaecological, $60(18 \%)$ of H\&N, $81(21 \%)$ of thoracic, and 42 (9\%) of urological cancers). The median surgery time was $155 \mathrm{~min}$ for all cancers, while the median theatre time was $226 \mathrm{~min}$. Major complications (Clavien Dindo Classification III or higher) were recorded for 3\% $(n=49)$. Readmissions were required for $3 \%(23(9 \%)$ for gynaecological, $14(4 \%)$ for $\mathrm{H} \& \mathrm{~N}, 2(<1 \%)$ for thoracic, and $2(<1 \%)$ for urological cancers). ICU stay of more than $24 \mathrm{~h}$ was required for $5 \%(n=78)$ of all cancer patients $(8(3 \%)$ of gynaecological, $1(<1 \%)$ for $\mathrm{H} \& \mathrm{~N}, 68(18 \%)$ of thoracic, and $1(<1 \%)$ for urological cancers.

Nine $(1 \%)$ patients developed COVID-19 post-operatively $(1(<1 \%)$ of gynaecological, $7(2 \%)$ of $\mathrm{H} \& \mathrm{~N}$, and $1(<1 \%)$ of urological cancer); of these, only the gynaecological patient went on to develop severe disease and died from COVID-19. Death from other causes was seen in $11(1 \%)$ of cancer patients $(2(<1 \%)$ of gynaecological, $3(1 \%)$ of $H \& N$, and $6(2 \%)$ of thoracic cancers). Table 3 summarises COVID-19 outcomes in IEO patients.

Figure 1a illustrates the number of weekly COVID-19 cases in Milan and surgeries per week for 1 March to 30 September 2020, as well as the comparable period in 2019. There was no constant marked decline in the number of surgeries performed in 2020 compared to the same period in 2019, except for weeks 27 to 32 where less surgeries were performed in 2020. The number of COVID-19 cases had a steep rise during the first four weeks observed, reaching 3545 cases in week 13 (15-21 March). Subsequently, the number of weekly COVID-19 cases began to gradually decrease with the exception of a second smaller peak in week 18 (2546 cases from 20th to 26th March). However, from week 35 onwards, cases began to moderately rise again. 


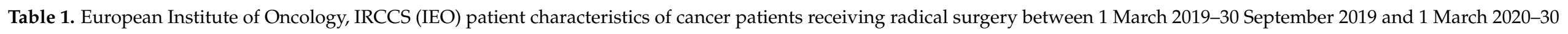
September 2020, divided by cancer site.

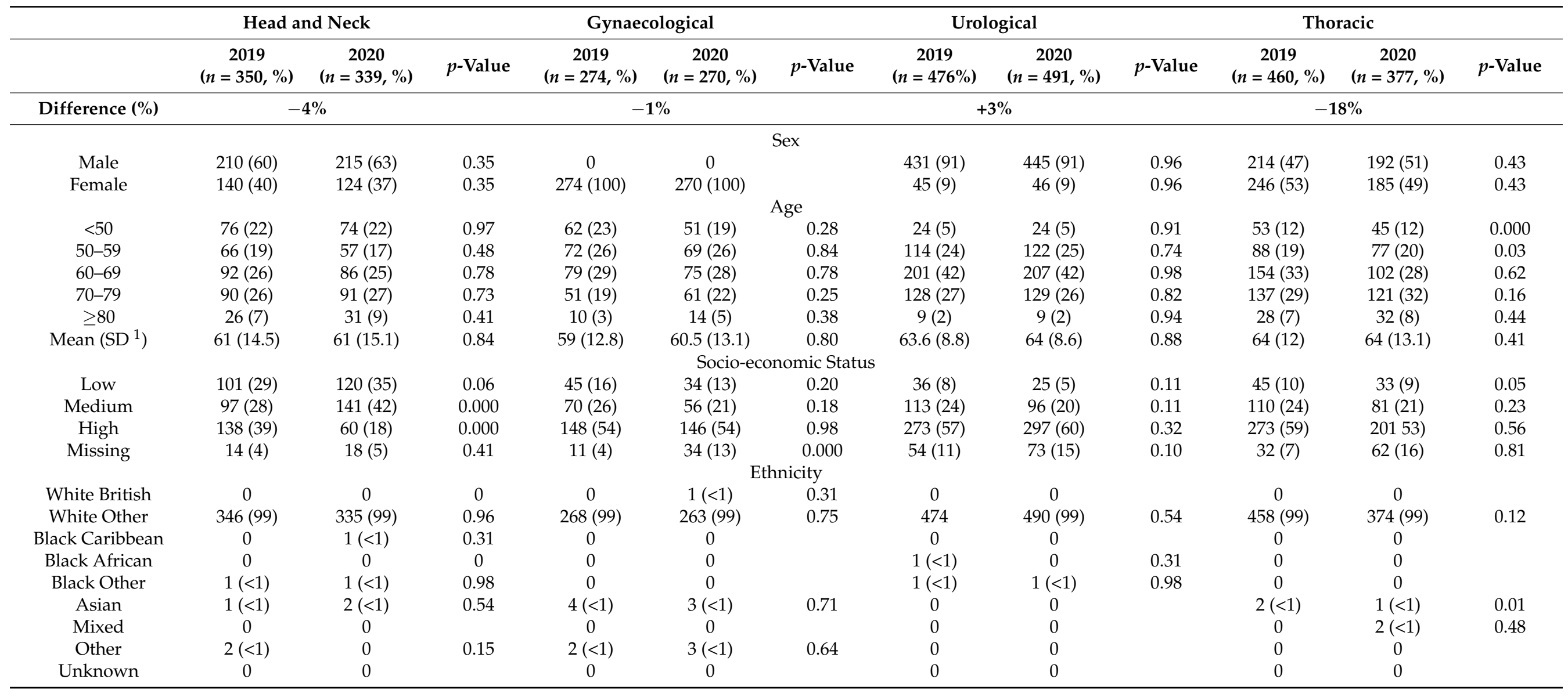


Table 1. Cont.

\begin{tabular}{|c|c|c|c|c|c|c|c|c|c|c|c|c|}
\hline & \multicolumn{2}{|c|}{ Head and Neck } & \multicolumn{4}{|c|}{ Gynaecological } & \multicolumn{2}{|c|}{ Urological } & \multicolumn{4}{|c|}{ Thoracic } \\
\hline & $\begin{array}{c}2019 \\
(n=350, \%)\end{array}$ & $\begin{array}{c}2020 \\
(n=339, \%)\end{array}$ & $p$-Value & $\begin{array}{c}2019 \\
(n=274, \%)\end{array}$ & $\begin{array}{c}2020 \\
(n=270, \%)\end{array}$ & $p$-Value & $\begin{array}{c}2019 \\
(n=476 \%)\end{array}$ & $\begin{array}{c}2020 \\
(n=491, \%)\end{array}$ & $p$-Value & $\begin{array}{c}2019 \\
(n=460, \%)\end{array}$ & $\begin{array}{c}2020 \\
(n=377, \%)\end{array}$ & $p$-Value \\
\hline Difference (\%) & \multicolumn{2}{|c|}{$-4 \%$} & \multicolumn{4}{|c|}{$-1 \%$} & \multicolumn{2}{|c|}{$+3 \%$} & \multicolumn{4}{|c|}{$-18 \%$} \\
\hline \multicolumn{13}{|c|}{ Co-morbidities } \\
\hline Hypertension & $141(40)$ & $113(33)$ & 0.05 & $74(27)$ & $76(28)$ & 0.76 & 184 & $180(37)$ & 0.52 & $167(36)$ & $130(34)$ & 0.06 \\
\hline Diabetes Mellitus & $27(8)$ & $35(10)$ & 0.23 & $27(10)$ & $8(<1)$ & 0.000 & $39(39)$ & $36(7)$ & 0.61 & $45(10)$ & $15(4)$ & 0.76 \\
\hline Lung Conditions & $26(7)$ & $24(7)$ & 0.85 & $11(4)$ & $6(<1)$ & 0.22 & $21(5)$ & $18(4)$ & 0.55 & $34(8)$ & $43(11)$ & 0.61 \\
\hline Renal Impairment & $8(2)$ & $5(1)$ & 0.43 & $5(2)$ & $1(<1)$ & 0.10 & $7(1)$ & $11(2)$ & 0.37 & $14(3)$ & $2(<1)$ & 0.72 \\
\hline Liver Conditions & $11(3)$ & $6(2)$ & 0.24 & $6(2)$ & $3(<1)$ & 0.32 & $11(2)$ & $13(3)$ & 0.73 & $8(2)$ & $2(<1)$ & 0.55 \\
\hline $\mathrm{CVD}^{2}$ & $57(16)$ & $63(19)$ & 0.42 & $30(11)$ & $24(9)$ & 0.42 & $82(17)$ & $74(15)$ & 0.36 & $95(21)$ & $62(16)$ & 0.52 \\
\hline \multicolumn{13}{|c|}{ Performance status } \\
\hline 0 & $182(52)$ & $212(63)$ & 0.004 & $239(87)$ & $261(97)$ & 0.000 & NA & NA & & NA & NA & \\
\hline 1 & $162(46)$ & $120(35)$ & 0.003 & $28(10)$ & $8(3)$ & 0.000 & NA & NA & & NA & NA & \\
\hline 2 & $5(<1)$ & $7(2)$ & 0.52 & $6(2)$ & 0 & 0.01 & NA & NA & & NA & NA & \\
\hline 3 & $1(<1)$ & 0 & 0.31 & $1(<1)$ & $1(<1)$ & 0.99 & NA & NA & & NA & NA & \\
\hline 4 & 0 & 0 & & 0 & 0 & & NA & NA & & NA & NA & \\
\hline
\end{tabular}

${ }^{1}$ Standard deviation, ${ }^{2}$ Cardiovascular disease. 
Table 2. Surgical outcomes of IEO and SELCA patients receiving radical treatment from between 1 March 2020-30 September 2020 and 23 March $2020-8$ September 2020.

\begin{tabular}{|c|c|c|c|c|c|c|c|c|c|c|}
\hline & $\begin{array}{l}\text { Breast } \\
(n, \%)\end{array}$ & $\begin{array}{c}\text { Colorectal } \\
(n, \%)\end{array}$ & $\begin{array}{c}\text { Gynaecological } \\
(n, \%)\end{array}$ & $\begin{array}{c}\text { Head and Neck } \\
(n, \%)\end{array}$ & $\begin{array}{l}\text { Liver } \\
(n, \%)\end{array}$ & $\begin{array}{l}\text { Plastics } \\
(n, \%)\end{array}$ & $\begin{array}{c}\text { Thoracic } \\
(n, \%)\end{array}$ & $\begin{array}{c}\text { Upper } \\
\text { Gastrointestinal } \\
(n, \%)\end{array}$ & $\begin{array}{c}\text { Urology } \\
(n, \%)\end{array}$ & $\begin{array}{l}\text { Total } \\
(n, \%)\end{array}$ \\
\hline \multicolumn{11}{|c|}{ IEO } \\
\hline ASA $^{1}$ grade III/IV/V & & & $24(9)$ & $60(18)$ & & & $81(21)$ & & $42(9)$ & $207(14)$ \\
\hline Surgery time-mins & & & 191 & 112 & & & 101 & & 214 & 155 \\
\hline (Median, IQR ${ }^{2}$ ) & & & $(28-658)$ & $(8-777)$ & & & $(54-161)$ & & $(70-525)$ & $(23-687)$ \\
\hline Theatre time-mins & & & 274 & 163 & & & 178 & & 288 & 226 \\
\hline $\mathrm{ICU}^{3}$ stay $>24 \mathrm{~h}$ & & & $8(3)$ & $1(<1)$ & & & $68(18)$ & & $1(<1)$ & $78(5)$ \\
\hline Pneumonia & & & $6(2)$ & $4(1)$ & & & 0 & & $2(<1)$ & $12(1)$ \\
\hline $\operatorname{LOS}^{4}$-days & & & 4 & 4 & & & 5 & & 3 & 4 \\
\hline Re-admissions & & & $23(9)$ & $14(4)$ & & & $2(<1)$ & & $2(<1)$ & $41(3)$ \\
\hline Complications & & & $58(21)$ & $64(19)$ & & & $57(15)$ & & $43(9)$ & $222(15)$ \\
\hline $\mathrm{I}$ & & & $18(7)$ & $39(12)$ & & & $23(6)$ & & $12(2)$ & $92(6)$ \\
\hline II & & & $20(7)$ & $8(2)$ & & & $25(7)$ & & $28(6)$ & $81(6)$ \\
\hline IIIA & & & $5(2)$ & $1(<1)$ & & & $6(2)$ & & $1(<1)$ & $13(<1)$ \\
\hline IIIB & & & $8(3)$ & $14(4)$ & & & 0 & & $2(<1)$ & $24(2)$ \\
\hline IVB & & & 0 & $1(<1)$ & & & $1(<1)$ & & 0 & $2(<1)$ \\
\hline $\mathrm{V}$ & & & $2(1)$ & 0 & & & $1(<1)$ & & 0 & $3(<1)$ \\
\hline \multicolumn{11}{|c|}{ SELCA } \\
\hline Surgeries & $n=321$ & $n=129$ & $n=114$ & $n=152$ & $n=92$ & $n=56$ & $n=305$ & $n=72$ & $n=312$ & $n=1553$ \\
\hline ASA grade III/IV/V & $12(4)$ & $18(14)$ & $19(17)$ & $20(13)$ & $12(13)$ & $11(20)$ & $91(30)$ & $9(13)$ & $48(15)$ & $240(22)$ \\
\hline Surgery time - mins & 79 & 143 & 149 & 126 & 217 & 50 & 118 & 187 & 145 & 120 \\
\hline (Median, IQR) & $(55-106)$ & $(90-218)$ & $(100-192)$ & $(74-330)$ & $(159-320)$ & $(39-111)$ & $(85-148)$ & $(116-292)$ & $(55-190)$ & (73-183) \\
\hline Theatre time-mins & 140 & 237 & 232 & 194 & 309 & 112 & 195 & 290 & 201 & 195 \\
\hline (Median, IQR) & $(115-173)$ & $(175-332)$ & $(184-290)$ & $(141-445)$ & $(255-413)$ & $(69-175)$ & $(161-225)$ & $(208-411)$ & $(113-254)$ & $(138-263)$ \\
\hline ICU stay $>24 h$ & $19(6)$ & $50(39)$ & $3(18)$ & $47(31)$ & $68(74)$ & $1(2)$ & $152(50)$ & $44(61)$ & $42(13)$ & $155(11)$ \\
\hline Pneumonia & 0 & 0 & 0 & $2(1)$ & 0 & 0 & $52(17)$ & 0 & 0 & $55(6)$ \\
\hline LOS-days & 1 & 6 & 1 & 3 & 7 & 0 & 6 & 8 & 2 & 4 \\
\hline Re-admissions & $2(1)$ & $5(4)$ & 9 & $8(5)$ & $6(7)$ & $1(2)$ & $6(2)$ & 0 & $2(1)$ & $36(11)$ \\
\hline
\end{tabular}


Table 3. COVID-19 outcomes of IEO and SELCA patients receiving radical treatment from between 1 March 2020-30 September 2020 and 23 March 2020-8 September 2020.

\begin{tabular}{|c|c|c|c|c|c|c|c|c|c|c|}
\hline & $\begin{array}{c}\text { Breast } \\
n, \%\end{array}$ & $\begin{array}{c}\text { Colorectal } \\
n, \%\end{array}$ & $\begin{array}{c}\text { Gynaecological } \\
n, \%\end{array}$ & $\begin{array}{c}\text { Head and Neck } \\
n, \%\end{array}$ & $\begin{array}{l}\text { Liver } \\
n, \%\end{array}$ & $\begin{array}{c}\text { Plastics } \\
n, \%\end{array}$ & $\begin{array}{c}\text { Thoracic } \\
n, \%\end{array}$ & $\begin{array}{c}\text { Upper } \\
\text { Gastrointestinal } \\
n, \%\end{array}$ & $\begin{array}{c}\text { Urology } \\
n, \%\end{array}$ & $\begin{array}{l}\text { Total } \\
n, \%\end{array}$ \\
\hline \multicolumn{11}{|c|}{ IEO } \\
\hline \multicolumn{11}{|c|}{ COVID status } \\
\hline Negative & & & $176(65)$ & $332(98)$ & & & $288(76)$ & & $490(99)$ & $1286(87)$ \\
\hline Positive & & & $1(<1)$ & $7(2)$ & & & 0 & & $1(<1)$ & $9(1)$ \\
\hline Unknown & & & $93(34)$ & 0 & & & $89(24)$ & & 0 & $182(12)$ \\
\hline Mild and moderate & & & 0 & $7(2)$ & & & 0 & & $1(<1)$ & $8(<1)$ \\
\hline Severe & & & $1(<1)$ & 0 & & & 0 & & 0 & $1(<1)$ \\
\hline \multicolumn{11}{|c|}{ Death } \\
\hline All-cause (30 days) & & & $2(<1)$ & $3(1)$ & & & $6(2)$ & & 0 & $11(1)$ \\
\hline All-cause (90 days) & & & $1(<1)$ & 0 & & & 0 & & 0 & $1(<1)$ \\
\hline \multicolumn{11}{|c|}{ SELCA } \\
\hline Surgeries & $n=321$ & $n=129$ & $n=114$ & $n=152$ & $n=92$ & $n=56$ & $n=305$ & $n=72$ & $n=312$ & $n=1553$ \\
\hline \multicolumn{11}{|c|}{ COVID status } \\
\hline $\begin{array}{l}\text { Negative } \\
\text { Positive } \\
\text { Unknown }\end{array}$ & $1(<1)$ & 0 & $1(<1)$ & 4 & 0 & 0 & 0 & 0 & $1(<1)$ & $7(<1)$ \\
\hline \multicolumn{11}{|c|}{ Death } \\
\hline All-cause (30 days) & 0 & 0 & 0 & 0 & 0 & 0 & $4(1)$ & 0 & $2(1)$ & $6(<1)$ \\
\hline All-cause (90 days) & 0 & 0 & 0 & $2(1)$ & $2(2)$ & $1(2)$ & $11(4)$ & 0 & $5(2)$ & $21(1)$ \\
\hline
\end{tabular}



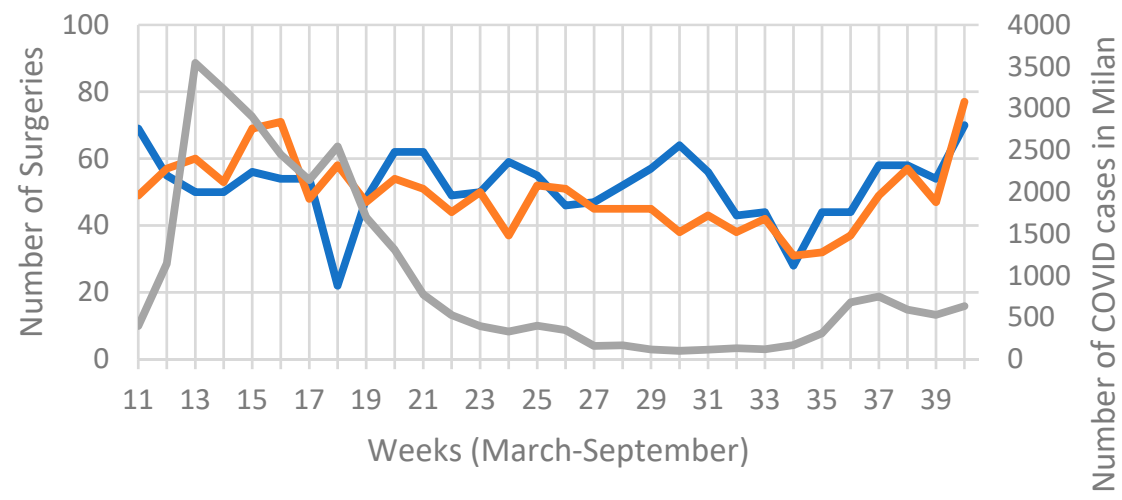

Surgeries Performed in 2019 Surgeries Performed in 2020 $\longrightarrow$ COVID-19 Cases in Milan

(a)

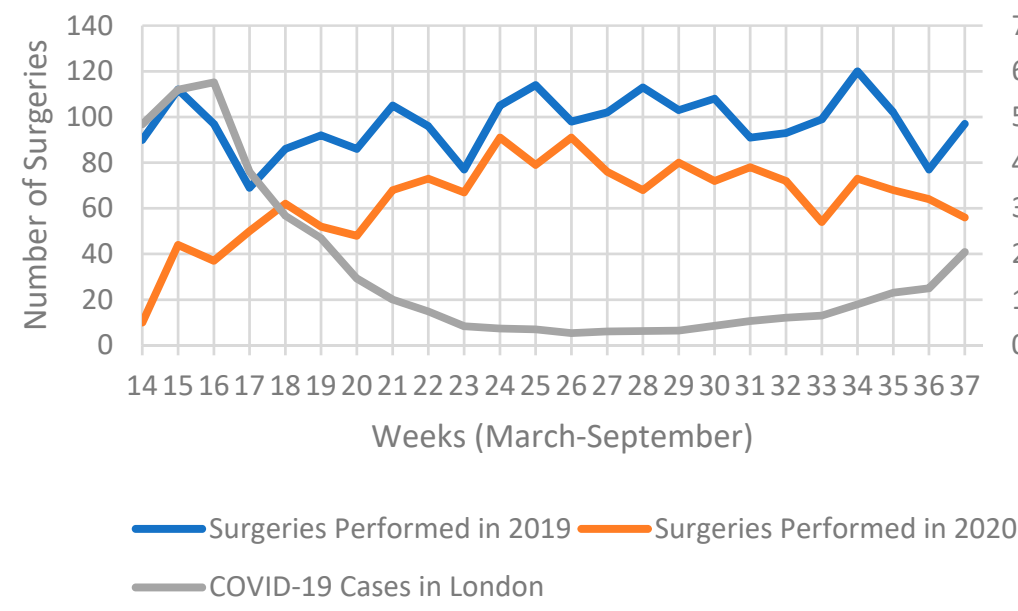

(b)

Figure 1. Chart illustrating: (a) weekly COVID-19 cases in Milan (and metropolitan area), and number of surgeries 1 March 2020-30 September 2020; (b) weekly SARS-CoV-2 (COVID-19) cases in London (and metropolitan area), and number of surgeries performed in South East London Cancer Alliance (SELCA) between 23 March 2019-8 September 2019 and 23 March 2020-8 September 2020.

\subsection{SELCA}

At SELCA centres in London, there was a decline of $34 \%$ radical surgeries performed from 23 March to 8 September 2020, compared to the same period in 2019. There were 1553 radical surgeries in the observed period (321 of breast, 129 of colorectal, 114 of gynaecological, 152 of H\&N, 92 of liver, 56 of plastics, 305 thoracic, 72 of upper gastrointestinal (GI), and 312 of urological cancers), compared to 2336 in 2019. The most notable declines were seen for plastic/skin surgeries, with a decline of $80 \%$ ( $n=56$ vs. $278, p=0.00)$; followed by colorectal with $59 \%$ ( $n=129$ vs. $310, p=0.00)$, and breast with $38 \%$ ( $n=321$ vs. 519 , $p=0.24)$. However, there was an increase in the number of $H \& N(9 \%, n=152$ vs. 139 , $p=0.00)$ and upper GI $(18 \%, n=72$ vs. $61, p=0.00)$ cancer surgeries in 2020 compared to 2019.

Clinical and demographic characteristics are shown in Tables 4, 5 and A1. Most of these characteristics were comparable between both periods, except for performance status, where more patients with lower performance status were operated on in 2020, compared to 2019 . 


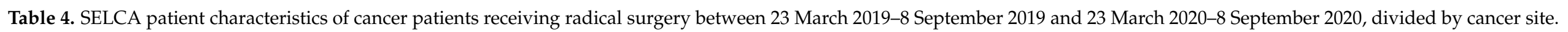

\begin{tabular}{|c|c|c|c|c|c|c|c|c|c|c|c|c|}
\hline \multirow[b]{3}{*}{ Difference (\%) } & \multicolumn{2}{|c|}{ Breast } & \multicolumn{4}{|c|}{ Colorectal } & \multicolumn{2}{|c|}{ Gynaecological } & \multicolumn{4}{|c|}{ Head and Neck } \\
\hline & $\begin{array}{c}2019 \\
(n=519, \%)\end{array}$ & $\begin{array}{c}2020 \\
(n=321, \%)\end{array}$ & $p$-Value & $\begin{array}{c}2019 \\
(n=310, \%)\end{array}$ & $\begin{array}{c}2020 \\
(n=129, \%)\end{array}$ & $p$-Value & $\begin{array}{c}2019 \\
(n=171, \%)\end{array}$ & $\begin{array}{c}2020 \\
(n=114, \%)\end{array}$ & $p$-Value & $\begin{array}{c}2019 \\
(n=139, \%)\end{array}$ & $\begin{array}{c}2020 \\
(n=152, \%)\end{array}$ & $p$-Value \\
\hline & \multicolumn{2}{|c|}{$-38 \%$} & \multicolumn{4}{|c|}{$-59 \%$} & \multicolumn{2}{|c|}{$-33 \%$} & \multicolumn{4}{|c|}{$+9 \%$} \\
\hline & & & & & Sex & & & & & & & \\
\hline Male & $6(1)$ & $6(2)$ & 0.42 & $174(56)$ & $71(55)$ & 0.83 & 0 & 0 & & $71(51)$ & $75(49)$ & 0.38 \\
\hline \multicolumn{13}{|c|}{ Age } \\
\hline$<50$ & $163(31)$ & $116(36)$ & 0.16 & $34(11)$ & $16(12)$ & 0.67 & $41(24)$ & $35(31)$ & 0.21 & $44(32)$ & $49(32)$ & 0.45 \\
\hline $50-59$ & $33(6)$ & $97(30)$ & 0.00 & $49(16)$ & $33(26)$ & 0.02 & $13(8)$ & $31(27)$ & 0.00 & $9(8)$ & $37(24)$ & 0.00 \\
\hline $60-69$ & $145(25)$ & $58(18)$ & 0.00 & $49(16)$ & $38(29)$ & 0.00 & $47(27)$ & $23(20)$ & 0.14 & $29(21)$ & $38(25)$ & 0.20 \\
\hline $70-79$ & $110(21)$ & $38(12)$ & 0.00 & $88(28)$ & $28(22)$ & 0.13 & $30(18)$ & $19(17)$ & 0.84 & $31(22)$ & $24(16)$ & 0.07 \\
\hline$\geq 80$ & $68(13)$ & $12(4)$ & 0.00 & $90(29)$ & $14(11)$ & 0.00 & $40(23)$ & $6(5)$ & 0.00 & $26(19)$ & $4(3)$ & 0.00 \\
\hline Mean $\left(\mathrm{SD}^{1}\right)$ & $56(13.3)$ & $54(12.9)$ & & $68(13.7)$ & $64(13.1)$ & & $59(14.8)$ & $57(14.4)$ & & $59(14.7)$ & $57(16.2)$ & \\
\hline \multicolumn{13}{|c|}{ Socioeconomic Status } \\
\hline Low & $101(19)$ & $59(18)$ & 0.69 & $44(14)$ & $20(16)$ & 0.72 & $32(19)$ & $15(13)$ & 0.20 & $22(16)$ & $27(18)$ & 0.32 \\
\hline Medium & $289(56)$ & $171(54)$ & 0.49 & $118(39)$ & $73(57)$ & 0.00 & $93(54)$ & $66(58)$ & 0.55 & $76(55)$ & $77(51)$ & 0.24 \\
\hline High & $108(21)$ & $91(28)$ & 0.01 & $88(28)$ & $36(27)$ & 0.91 & $46(27)$ & $33(29)$ & 0.70 & $39(28)$ & $47(31)$ & 0.29 \\
\hline \multicolumn{12}{|c|}{ Ethnicity } & 0.25 \\
\hline White British & $165(32)$ & $91(28)$ & 0.28 & $86(28)$ & $34(26)$ & 0.76 & $49(29)$ & $34(30)$ & 0.83 & $72(53)$ & $58(38)$ & 0.009 \\
\hline White Other & $52(10)$ & $42(13)$ & 0.18 & $25(8)$ & $10(8)$ & 0.91 & $22(13)$ & $17(15)$ & 0.62 & $15(11)$ & $14(9)$ & 0.32 \\
\hline Black Caribbean & $28(5)$ & $18(6)$ & 0.89 & $4(1)$ & $1(<1)$ & 0.60 & $2(1)$ & $2(2)$ & 0.69 & $5(4)$ & $2(1)$ & 0.10 \\
\hline Black African & $16(3)$ & $12(4)$ & 0.61 & $8(3)$ & 0 & 0.00 & $5(3)$ & $6(5)$ & 0.34 & $3(2)$ & $3(2)$ & 0.45 \\
\hline Black Other & $28(5)$ & $17(5)$ & 0.95 & $2(1)$ & $4(3)$ & 0.12 & $5(3)$ & $6(5)$ & 0.34 & $1(<1)$ & $5(3)$ & 0.05 \\
\hline Asian & $23(4)$ & $11(3)$ & 0.45 & $2(1)$ & $4(3)$ & 0.12 & $5(3)$ & $2(2)$ & 0.51 & $9(6)$ & $7(5)$ & 0.24 \\
\hline Mixed & $15(3)$ & $11(3)$ & 0.66 & 0 & $2(2)$ & 0.15 & 0 & 0 & & $2(1)$ & 0 & 0.07 \\
\hline Other & $8(2)$ & $8(2)$ & 0.35 & $2(1)$ & 0 & 0.15 & $4(2)$ & $3(2)$ & 0.87 & $1(<1)$ & $2(1)$ & 0.30 \\
\hline Unknown & $184(35)$ & $111(35)$ & 0.79 & $181(57)$ & $74(57)$ & 0.84 & $79(46)$ & $44(39)$ & 0.20 & $31(22)$ & $61(41)$ & 0.00 \\
\hline \multicolumn{13}{|c|}{ Comorbidities } \\
\hline Hypertension & $57(11)$ & $20(6)$ & 0.01 & $29(9)$ & $2(2)$ & 0.00 & $53(31)$ & $10(9)$ & 0.00 & $46(33)$ & $3(2)$ & 0.00 \\
\hline Diabetes Mellitus & $25(5)$ & $11(3)$ & 0.31 & $17(5)$ & $8(6)$ & 0.77 & $27(16)$ & $6(5)$ & 0.00 & $22(56)$ & $1(<1)$ & 0.00 \\
\hline Lung Conditions & $4(1)$ & $6(2)$ & 0.19 & $24(8)$ & $6(5)$ & 0.19 & $3(2)$ & $3(3)$ & 0.62 & $7(5)$ & $4(3)$ & 0.14 \\
\hline Renal Impairment & $8(2)$ & 0 & 0.00 & $17(5)$ & $2(2)$ & 0.01 & $14(8)$ & $1(<1)$ & 0.00 & $5(4)$ & 0 & 0.01 \\
\hline Liver Conditions & $1(<1)$ & 0 & 0.31 & $9(3)$ & $2(2)$ & 0.34 & $3(2)$ & 0 & 0.08 & $1(<1)$ & $1(<1)$ & 0.47 \\
\hline $\mathrm{CVD}^{2}$ & $4(1)$ & $6(2)$ & 0.19 & $8(3)$ & $11(9)$ & 0.02 & $9(5)$ & $1(<1)$ & 0.02 & $8(6)$ & $2(1)$ & 0.02 \\
\hline
\end{tabular}


Table 4. Cont

\begin{tabular}{|c|c|c|c|c|c|c|c|c|c|c|c|c|}
\hline \multirow[b]{3}{*}{ Difference (\%) } & \multicolumn{2}{|c|}{ Breast } & \multicolumn{4}{|c|}{ Colorectal } & \multicolumn{2}{|c|}{ Gynaecological } & \multicolumn{4}{|c|}{ Head and Neck } \\
\hline & $\begin{array}{c}2019 \\
(n=519, \%)\end{array}$ & $\begin{array}{c}2020 \\
(n=321, \%)\end{array}$ & $p$-Value & $\begin{array}{c}2019 \\
(n=310, \%)\end{array}$ & $\begin{array}{c}2020 \\
(n=129, \%)\end{array}$ & $p$-Value & $\begin{array}{c}2019 \\
(n=171, \%)\end{array}$ & $\begin{array}{c}2020 \\
(n=114, \%)\end{array}$ & $p$-Value & $\begin{array}{c}2019 \\
(n=139, \%)\end{array}$ & $\begin{array}{c}2020 \\
(n=152, \%)\end{array}$ & $p$-Value \\
\hline & \multicolumn{2}{|c|}{$-38 \%$} & \multicolumn{4}{|c|}{$-59 \%$} & \multicolumn{2}{|c|}{$-33 \%$} & \multicolumn{4}{|c|}{$+9 \%$} \\
\hline \multicolumn{13}{|c|}{ Performance status } \\
\hline 0 & $134(26)$ & $185(58)$ & 0.00 & $21(7)$ & $41(32)$ & 0.00 & $16(9)$ & $42(37)$ & 0.00 & $6(4)$ & $32(21)$ & 0.00 \\
\hline 1 & $134(26)$ & $81(25)$ & 0.84 & $48(15)$ & $40(31)$ & 0.00 & $85(50)$ & $52(46)$ & 0.49 & $41(34)$ & $49(32)$ & 0.30 \\
\hline 2 & $47(9)$ & 0 & 0.00 & $15(5)$ & $9(7)$ & 0.40 & $45(26)$ & $6(5)$ & 0.00 & $27(19)$ & $9(6)$ & 0.00 \\
\hline 3 & $2(<1)$ & $1(<1)$ & 0.85 & $2(1)$ & $1(<1)$ & 0.88 & $8(5)$ & $3(3)$ & 0.35 & $1(<1)$ & 0 & 0.15 \\
\hline Unknown & $188(36)$ & $19(6)$ & 0.00 & $224(72)$ & $38(29)$ & 0.00 & $16(9)$ & $7(6)$ & 0.30 & $64(27)$ & $62(41)$ & 0.18 \\
\hline
\end{tabular}

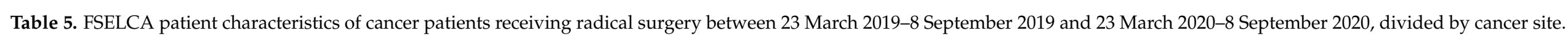

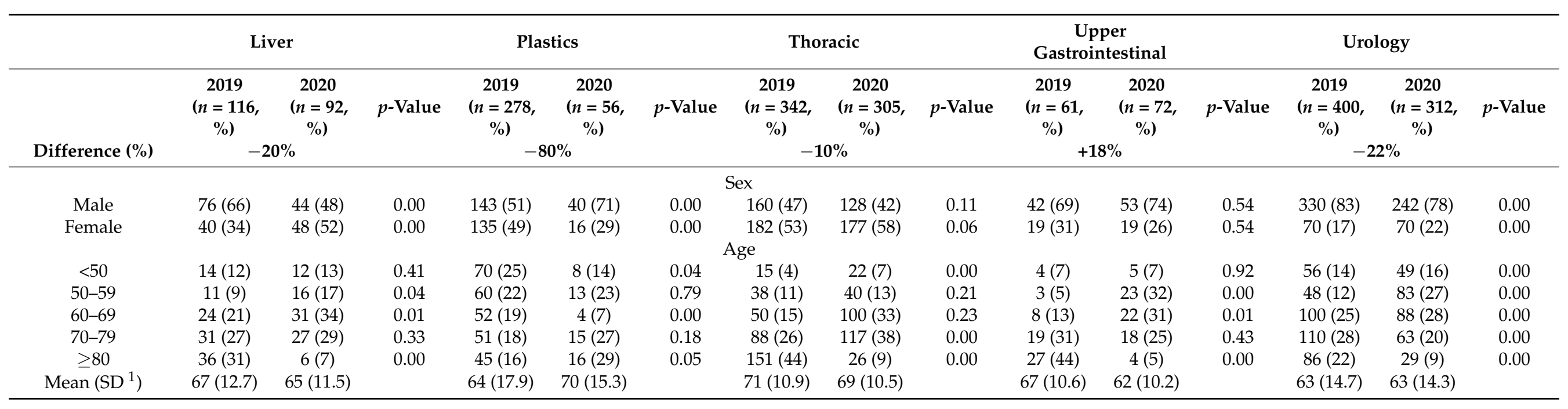


Table 5. Cont.

\begin{tabular}{|c|c|c|c|c|c|c|c|c|c|c|c|c|c|c|c|}
\hline \multirow[b]{3}{*}{ Difference (\%) } & \multicolumn{2}{|c|}{ Liver } & \multicolumn{3}{|c|}{ Plastics } & \multicolumn{4}{|c|}{ Thoracic } & \multicolumn{2}{|c|}{$\begin{array}{c}\text { Upper } \\
\text { Gastrointestinal }\end{array}$} & \multicolumn{4}{|c|}{ Urology } \\
\hline & $\begin{array}{c}2019 \\
(n=116 \\
\%)\end{array}$ & $\begin{array}{c}2020 \\
(n=92 \\
\%)\end{array}$ & $p$-Value & $\begin{array}{c}2019 \\
(n=278 \\
\%)\end{array}$ & $\begin{array}{c}2020 \\
(n=56 \\
\%)\end{array}$ & $p$-Value & $\begin{array}{c}2019 \\
(n=342 \\
\%)\end{array}$ & $\begin{array}{c}2020 \\
(n=305 \\
\%)\end{array}$ & $p$-Value & $\begin{array}{c}2019 \\
(n=61, \\
\%)\end{array}$ & $\begin{array}{c}2020 \\
(n=72 \\
\%)\end{array}$ & $p$-Value & $\begin{array}{c}2019 \\
(n=400 \\
\%)\end{array}$ & $\begin{array}{c}2020 \\
(n=312 \\
\%)\end{array}$ & $p$-Value \\
\hline & \multicolumn{2}{|c|}{$-20 \%$} & & \multicolumn{2}{|c|}{$-80 \%$} & \multicolumn{4}{|c|}{$-10 \%$} & \multicolumn{2}{|c|}{$+18 \%$} & \multicolumn{4}{|c|}{$-22 \%$} \\
\hline & & & & & & Socioeco & omic Status & & & & & & & & \\
\hline Low & $8(7)$ & $4(4)$ & 0.21 & $14(5)$ & $10(18)$ & 0.01 & $51(15)$ & 40 (13) & 0.40 & $11(18)$ & $10(14)$ & 0.51 & 48 (12) & 59 (19) & 0.00 \\
\hline Medium & $34(29)$ & $48(52)$ & 0.00 & $115(41)$ & $20(36)$ & 0.42 & $162(47)$ & $133(44)$ & 0.21 & $21(34)$ & $35(49)$ & 0.09 & $178(45)$ & $148(47)$ & 0.00 \\
\hline Missing & $42(36)$ & 0 & 0.00 & $42(15)$ & 0 & 0.00 & $2(1)$ & 0 & 0.82 & $2(3)$ & 0 & 0.15 & $23(6)$ & $4(1)$ & 0.00 \\
\hline \multicolumn{16}{|c|}{ Ethnicity } \\
\hline White British & $23(20)$ & $24(26)$ & 0.14 & $90(32)$ & $23(41)$ & 0.22 & $96(28)$ & $49(16)$ & 0.00 & $29(48)$ & $25(35)$ & 0.13 & $79(20)$ & $75(2)$ & 0.13 \\
\hline White Other & $5(4)$ & $1(1)$ & 0.06 & $17(6)$ & $3(5)$ & 0.82 & $17(5)$ & $16(5)$ & 0.82 & $5(8)$ & $4(6)$ & 0.55 & $24(6)$ & $28(9)$ & 0.14 \\
\hline Black Caribbean & $2(2)$ & $3(3)$ & 0.24 & $1(<1)$ & 0 & 0.31 & $1(<1)$ & $3(1)$ & 0.76 & $2(3)$ & 0 & 0.15 & $9(2)$ & $6(2)$ & 0.13 \\
\hline Black African & $1(1)$ & $1(1)$ & 0.43 & 0 & 0 & & $2(<1)$ & $2(1)$ & 0.86 & 0 & $3(4)$ & 0.07 & $10(3)$ & $8(3)$ & 0.02 \\
\hline Black Other & 0 & 0 & & $1(<1)$ & 0 & 0.31 & $3(<1)$ & $2(1)$ & 0.70 & $1(2)$ & $1(1)$ & 0.90 & $17(4)$ & $9(3)$ & 0.00 \\
\hline Asian & 0 & 0 & & 0 & 9 & 0.00 & 0 & $2(1)$ & & $2(3)$ & 0 & 0.15 & $5(1)$ & 0 & 0.04 \\
\hline Mixed & $3(3)$ & 0 & 0.03 & 0 & $1(2)$ & 0.31 & $2(<1)$ & 0 & 0.33 & 0 & $1(1)$ & 0.31 & $1(<1)$ & $2(<1)$ & 0.00 \\
\hline Unknown & $76(65)$ & $62(67)$ & 0.38 & $169(61)$ & $29(52)$ & 0.21 & $219(64)$ & $230(75)$ & 0.00 & $22(36)$ & $36(50)$ & 0.10 & $251(63)$ & $183(59)$ & 0.00 \\
\hline \multicolumn{16}{|c|}{ Comorbidities } \\
\hline Hypertension & $2(2)$ & $14(15)$ & 0.00 & $22(8)$ & $10(18)$ & 0.06 & $156(46)$ & $23(8)$ & 0.00 & $32(52)$ & 0 & 0.00 & $115(29)$ & $8(3)$ & 0.00 \\
\hline Diabetes Mellitus & $2(2)$ & $9(10)$ & 0.00 & $9(3)$ & $3(5)$ & 0.50 & $51(15)$ & $22(7)$ & 0.00 & $7(11)$ & 0 & 0.00 & $46(12)$ & $21(7)$ & 0.00 \\
\hline Lung Conditions & $1(1)$ & $18(20)$ & 0.00 & $4(1)$ & $3(5)$ & 0.20 & 0 & $32(10)$ & & $7(11)$ & $2(3)$ & 0.05 & $9(2)$ & $14(4)$ & 0.00 \\
\hline Renal Impairment & 0 & 0 & & $3(1)$ & 0 & 0.08 & $25(7)$ & $1(<1)$ & 0.00 & $3(5)$ & 0 & 0.07 & $67(17)$ & $3(1)$ & 0.00 \\
\hline Liver Conditions & 0 & $5(5)$ & 0.01 & 0 & 0 & & $5(1)$ & 0 & 0.12 & $4(7)$ & 0 & 0.03 & $6(2)$ & 0 & 0.16 \\
\hline $\mathrm{CVD}^{2}$ & $1(1)$ & $22(24)$ & 0.00 & $5(2)$ & $10(18)$ & 0.00 & $33(10)$ & $37(12)$ & 0.08 & $4(7)$ & 0 & 0.03 & $13(3)$ & $21(7)$ & 0.00 \\
\hline \multicolumn{16}{|c|}{ Performance status } \\
\hline 0 & $1(1)$ & $47(51)$ & 0.00 & $2(1)$ & $19(34)$ & 0.00 & $13(4)$ & $62(20)$ & 0.21 & $1(2)$ & $8(11)$ & 0.01 & $85(21)$ & $146(47)$ & 0.00 \\
\hline 1 & $8(7)$ & $18(20)$ & 0.00 & $5(2)$ & $4(7)$ & 0.13 & 47 (14) & $75(25)$ & 0.00 & $15(25)$ & $26(36)$ & 0.14 & 69 (17) & $46(15)$ & 0.00 \\
\hline 2 & $2(2)$ & $14(15)$ & 0.00 & $6(2)$ & $4(7)$ & 0.16 & $10(3)$ & $11(4)$ & 0.42 & $5(8)$ & $3(4)$ & 0.34 & $21(5)$ & $13(4)$ & 0.00 \\
\hline 3 & 0 & 0 & & 0 & $1(2)$ & 0.31 & $2(<1)$ & 0 & 0.33 & 0 & 0 & & $1(<1)$ & $2(<1)$ & 0.15 \\
\hline 4 & 0 & 0 & & 0 & $1(2)$ & 0.31 & $1(<1)$ & 0 & 0.49 & 0 & 0 & & 0 & 0 & 0.5 \\
\hline Unknown & 105 (91) & $13(14)$ & & $265(95)$ & $27(48)$ & 0.00 & $269(79)$ & $157(51)$ & 0.00 & $40(65)$ & $35(49)$ & 0.04 & $224(56)$ & $105(34)$ & 0.00 \\
\hline
\end{tabular}


Surgical outcomes for SELCA patients 23 between March and 8 September 2020 are summarised in Table 2. A total of 240 patients (22\%) had an ASA grade of III or higher (12 (4\%) of breast, $18(14 \%)$ of colorectal, 19 (17\%) of gynaecological, $20(13 \%)$ of H\&N, $12(13 \%)$ of liver, 11 (20\%) of plastic, 91 (30\%) of thoracic, $9(13 \%)$ of upper GI, and $48(15 \%)$ of urological cancers. Median surgery and theatre times were 120 and $195 \mathrm{~min}$, respectively for all cancers. A total of $36(11 \%)$ of cancer patients required readmission (2 (1\%) of breast, $5(4 \%)$ of colorectal, $8(5 \%)$ of H\&N, $6(7 \%)$ of liver, $1(2 \%)$ of plastic, $6(2 \%)$ of thoracic, and $2(1 \%)$ of urological cancers). As for time in the ICU, $155(11 \%)$ of cancer patients required stays of more than $24 \mathrm{~h}(19(6 \%)$ of breast, 50 (39\%) of colorectal, $3(18 \%)$ of gynaecological, $47(31 \%)$ of H\&N, $68(74 \%)$ of liver, 1 (2\%) of plastic, $152(50 \%)$ of thoracic, $44(61 \%)$ of upper GI, and $42(13 \%)$ of urological cancers). A total of $55(6 \%)$ of patients developed pneumonia, and 52 (17\%) were from thoracic surgery.

Of the total of patient undergoing radical surgery, $7(<1 \%)$ developed COVID-19 $(1(<1 \%)$ of breast, $1(<1 \%)$ of gynaecological, $4(3 \%)$ of $H \& N$, and $1(<1 \%)$ of urological cancers. No patients died from COVID-19 complications. A total of $27(2 \%)$ of patients died of other causes $(2(1 \%)$ of H\&N, $2(2 \%)$ of liver, $1(2 \%)$ of plastic, $15(5 \%)$ of thoracic, and $7(3 \%)$ of urological cancers), where only $6(<1 \%)$ died within 30 days. COVID-19 outcomes for cancer patients undergoing radical surgery are summarised in Table 3.

Figure $1 b$ illustrates the number of weekly COVID-19 cases in London and surgeries per week for 23rd March to 8th September 2020, as well as the comparable period in 2019. There was a significant decrease in the number of surgeries throughout the observed period. The biggest difference was seen in the first 3 weeks analysed (23 March to 12 April). During these first three weeks observed, the number of COVID-19 cases were at their highest, reaching 5760 cases in week 16 (6-12 March). The number of surgeries in 2020 began to rise from week 17 (13 April); however, they never reached the same number as 2019. On the other hand, COVID-19 cases began to decline from week 17 onwards and maintained a plateau until cases began to rise again starting week 30 (13 July 2020).

\section{Discussion}

The IEO in Milan and SELCA hospitals in London were both at the epicentre of the first COVID-19 wave. While a decline of $6 \%$ and $34 \%$ in number of surgeries was observed in Milan and London, respectively, the current study has shown that the implemented COVID19 minimal pathways are safe for cancer patients requiring radical treatment. Even with different geographical setting, patient characteristics were comparable between both cancer hubs except for performance status, where in the SELCA population, more surgeries were performed in patients with a lower performance status ( 0 and 1$)$. This may have been to avoid patients with higher risk of developing severe COVID-19 disease and complications from getting infected. Regarding COVID-19 and surgical outcomes, readmissions in IEO were required for 3\% $(n=41)$ and 3\% $(n=59)$ had major complications. Less than $1 \%(n=9)$ developed COVID-19, of which $80 \%(n=7)$ were H\&N cancers and had a mild/moderate disease. Only one gynaecological cancer patient had severe disease and died. In SELCA, readmissions were required for $11 \%(n=36)$ and $<1 \%(n=7)$ developed COVID-19 in the post-operative period. Details on the severity and/or death from COVID-19 were not reported. Only $2 \%(n=27)$ died from any cause.

Regardless of the high increase in the number of COVID-19 cases, the IEO saw a small decline in oncological surgery overall. Similar results were reported by Maspero et al. where cancer surgery was prioritised and remained stable, while overall surgical activity saw up to an $84 \%$ reduction in volume, both with minimal mortality due to COVID-19 [19]. In the IEO population, the biggest decline in number of surgeries was seen for thoracic cancers $(18 \%, n=377$ vs. 460$)$, this may be due to more restrictions applied in the selection of patients with underlying lung conditions, as these patients have been reported to have an increased risk of developing severe COVID-19 disease [20]. Moreover, a decline in the number of surgeries may reflect a gap in the early diagnosis of cancer due to COVID-19. On 
the other hand, the number of urological surgeries increased by $3 \%$ ( $n=491$ vs. 476) in 2020 compared to 2019; this was not in line with previous studies, where urological surgeries overall (including oncological surgeries) had a decline in volume of surgeries during the COVID-19 pandemic [21,22]. The IEO was chosen by the region as a reference centre for other non-operative hospitals for pandemic urgency. Many urological cancer patients were sent from these centres to the IEO, and this may explain the increase in this number for this division. In addition, the pathway undertaken by the IEO in Milan is similar to other pathways described in other Italian centres, which have had similar favourable results [23].

In the UK, cancer services and especially aggressive tumour types were given high priority to continue their activity (i.e., thoracic, H\&N, and upper GI) [24]. In our SELCA population, overall, oncological surgery had a decline of $34 \%(n=1553$ vs. 2336) in 2020 compared with 2019. The largest declines were seen for plastic/skin (80\%, $n=56$ vs. 278$)$, colorectal $(59 \%, n=129$ vs. 310$)$, and breast $(38 \%, n=321$ vs. 519$)$ cancer, while H\&N and upper GI had an increase of $9 \%$ and $18 \%$, respectively ( $n=152$ vs. 139, $n=72$ vs. 61 ). The increase in upper GI surgeries may be explained by an increase in minimally invasive surgeries for cancer patients and by a higher number of emergency procedures (bleeding/perforation) performed in the upper GI tract $[25,26]$. The large decrease in number of breast surgeries is likely due to delayed breast cancer diagnoses and a lower number of breast screening tests performed during this period. Similar results were seen in another observational study [27]. Moreover, the large decrease in number of surgeries for "non-urgent" cancer may have been due to the outbalance of high risk of new contagions compared with the risk of cancer progression in many cases. Similar results were reported by various cancer centres where surgeries were cancelled or postponed for months [24,28]. However, after the first wave of the pandemic, the number of cancer surgeries began to rise gradually [29]. Additionally, several studies have now reported on safe pathways to perform "non-urgent" cancer surgeries with minimal complications due to COVID-19 [30-33].

Overall, in both cancer hubs, the decision-making for the surgical prioritisation was individually reviewed by a panel of clinicians and dedicated virtual tumour board, and treatment plans were personalised taking into account patient comorbidities, performance status, tumour characteristics, availability of other oncological treatments, etc. The risk and benefits of each surgical procedure should be thoroughly weighed against the potential spread of COVID-19 disease [34]. Thus, an individualised approach is imperative in the treatment of cancers during the COVID-19 pandemic [18]. Moreover, the cancellation and postponement of elective and "non-urgent" cancer surgeries has created a backlog of patients who were planned to undergo radical treatment; this has great implications for both patients and healthcare providers [23]. It is critical for more cancer centres and specialties to begin to implement similar pathways in their institutes to reintroduce elective cancer surgery to prevent unnecessary delays in patient care.

Alarming results were reported by a large international survey that highlighted insufficient pre-operative screening of COVID-19 in the current surgical practice $[28,35,36]$. In addition, this survey found several discrepancies on the various pathways implemented across cancer centres. Thus, it is important to further inform future clinical guidelines to install a universal safe pathway to treat cancer patients. Further epidemiological studies are needed comparing data for more cancer sites, as well as cancer types and types of surgeries performed to have a more detailed look into the safest pathways for cancer patients. In addition, it is important for future studies to describe criteria used for choosing patients who are apt to undergo oncological surgery. Lastly, studies on the effects of surgical cancellation, on both patients and healthcare staff, are much needed to analyse the true effect the COVID-19 pandemic had on cancer care.

To our knowledge, the current study is among the first large cohorts comparing safe pathways for oncological surgery implemented in various hospitals in Milan and southeast London. Both of these cities were among the hardest-hit cities in Europe, and thus, they were among the first centres to implement COVID-19 minimal pathways. One of 
the major limitations of our study was the lack of data on COVID-19 severity status for SELCA patients.

\section{Conclusions}

Our findings suggest that although cancer patients have been previously identified as high risk from COVID-19, the implemented COVID-19 minimal pathways described here are safe for patients who require radical treatment. The implementation of hospital prevention plans designed to avoid the entry of COVID-19 positive patients and healthcare professionals are essential to safeguard delicate oncologic patients already hospitalised, the health personnel, and to be able to continue the life-saving activity of cancer centres. It is critical for all elective cancer surgeries to go back to their normal levels of functioning to avoid future complications due to delays in oncological care.

Author Contributions: Conceptualisation, M.V.H.; data acquisition, M.J.M.-I., M.T., B.R., C.M., S.I., J.O., I.M.F.C., A.S., S.M., R.N. (Rahul Nath), A.B., R.S. (Ricard Simo), C.T., P.S. (Parthi Srinivasan), A.P., A.D. (Andrew Davies), J.G., S.F., T.R., R.M., E.D., B.C., R.N. (Raj Nair), M.H., R.S. (Rosaria Scarpinata), P.S. (Paolo Sorelli), S.D., F.A.M., G.M., M.C., A.A., A.D. (Andrea Dell'Acqua), D.S., S.Z., D.R.D.S., D.B., R.B., G.P., A.M., O.d.C., L.S., M.A., F.M., S.G., U.J., H.H., K.H., and M.V.H..; data curation, H.D., G.R., R.D.B., M.J.M.-I. and M.T.; methodology, M.J.M.-I., M.T., and M.V.H.; formal analysis, M.J.M.-I.; supervision, M.V.H.; writing-original draft preparation, M.J.M..I. and M.T.; writing-review and editing, M.J.M.-I., M.T., B.R., C.M., S.I., J.O., I.M.F.C., A.S., S.M., R.N. (Rahul Nath), A.B., R.S. (Ricard Simo), C.T., P.S. (Parthi Srinivasan), A.P., A.D. (Andrew Davies), J.G., S.F., T.R., R.M., E.D., B.C., R.N. (Raj Nair), M.H., R.S. (Rosaria Scarpinata), P.S. (Paolo Sorelli), S.D., F.A.M., G.M., M.C., A.A., A.D. (Andrea Dell'Acqua), D.S., S.Z., D.R.D.S., D.B., R.B., G.P., A.M., O.d.C., L.S., M.A., F.M., S.G., U.J., H.H., K.H., and M.V.H. All authors have read and agreed to the published version of the manuscript.

Funding: This work was partially supported by the Italian Ministry of Health with Ricerca Corrente and $5 \times 1000$ funds.

Institutional Review Board Statement: In Data collection was approved by the European Institute of Oncology Ethics Committee (code. IEO 2432); and Guy's Cancer Cohort (Reference number: 18/NW/0297).

Data Availability Statement: The data presented in this study are available on request from the corresponding author. The data are not publicly available due to ethical reasons.

Conflicts of Interest: The authors declare no conflict of interest.

\section{Appendix A}

Table A1. Contains the patient characteristics of all cancer patients receiving radical surgery between 1 March 2019-30 September 2019 and 1 March 2020-30 September 2020 at the IEO, and 23 March 2019-8 September 2019 and 23 March 2020-8 September 2020 at SELCA.

\begin{tabular}{|c|c|c|c|c|c|c|}
\hline \multirow{3}{*}{ Difference (\%) } & \multicolumn{2}{|c|}{ IEO } & \multicolumn{4}{|c|}{ SEL Cancer Alliance } \\
\hline & $\begin{array}{c}2019 \\
(n=1560, \%)\end{array}$ & $\begin{array}{c}2020 \\
(n=1477, \%)\end{array}$ & $p$-Value & $\begin{array}{c}2019 \\
(n=2336, \%)\end{array}$ & $\begin{array}{c}2020 \\
(n=1553, \%)\end{array}$ & $p$-Value \\
\hline & \multicolumn{2}{|c|}{$-6 \%$} & \multicolumn{4}{|c|}{$-34 \%$} \\
\hline \multicolumn{7}{|c|}{ Sex } \\
\hline Male & 855 (55) & $852(58)$ & 0.11 & $1003(43)$ & $659(42)$ & 0.75 \\
\hline Female & 705 (45) & $625(42)$ & 0.11 & $1333(57)$ & $894(58)$ & 0.75 \\
\hline \multicolumn{7}{|c|}{ Age } \\
\hline$<50$ & $215(14)$ & $194(13)$ & 0.60 & 441 (19) & $312(20)$ & 0.35 \\
\hline 50-59 & $340(22)$ & $325(22)$ & 0.88 & $264(11)$ & $373(24)$ & 0.000 \\
\hline $60-69$ & $526(34)$ & 470 (32) & 0.26 & $504(22)$ & $402(26)$ & 0.002 \\
\hline $70-79$ & $406(26)$ & $402(32)$ & 0.45 & $558(24)$ & 349 (23) & 0.30 \\
\hline$\geq 80$ & $73(5)$ & $86(6)$ & 0.15 & $569(24)$ & $117(7)$ & 0.000 \\
\hline
\end{tabular}


Table A1. Cont.

\begin{tabular}{|c|c|c|c|c|c|c|}
\hline \multirow[b]{3}{*}{ Difference (\%) } & \multicolumn{2}{|c|}{ IEO } & \multicolumn{4}{|c|}{ SEL Cancer Alliance } \\
\hline & $\begin{array}{c}2019 \\
(n=1560, \%)\end{array}$ & $\begin{array}{c}2020 \\
(n=1477, \%)\end{array}$ & $p$-Value & $\begin{array}{c}2019 \\
(n=2336, \%)\end{array}$ & $\begin{array}{c}2020 \\
(n=1553, \%)\end{array}$ & $p$-Value \\
\hline & \multicolumn{2}{|c|}{$-6 \%$} & \multicolumn{4}{|c|}{$-34 \%$} \\
\hline \multicolumn{7}{|c|}{ Socioeconomic Status } \\
\hline Low & $227(15)$ & $212(14)$ & 0.87 & $331(14)$ & $244(16)$ & 0.18 \\
\hline Medium & $390(25)$ & $374(25)$ & 0.83 & $1086(46)$ & $771(49)$ & 0.05 \\
\hline High & $832(53)$ & $704(48)$ & 0.001 & $725(31)$ & $533(34)$ & 0.03 \\
\hline Missing & $111(7)$ & $187(13)$ & 0.000 & $194(8)$ & $5(1)$ & 0.00 \\
\hline \multicolumn{7}{|c|}{ Ethnicity } \\
\hline White British & 0 & 1 & 0.31 & $689(29)$ & $413(27)$ & 0.04 \\
\hline White Other & $1546(99)$ & $1462(99)$ & 0.73 & $182(8)$ & $135(9)$ & 0.31 \\
\hline Black Caribbean & 0 & $1(<1)$ & 0.31 & $54(2)$ & $35(2)$ & 0.90 \\
\hline Black African & $1(<1)$ & 0 & 0.31 & $45(2)$ & $35(2)$ & 0.48 \\
\hline Black Other & $2(<1)$ & $2(<1)$ & 0.95 & $58(2)$ & $44(3)$ & 0.50 \\
\hline Asian & $7(<1)$ & $6(<1)$ & 0.85 & $46(2)$ & $26(2)$ & 0.49 \\
\hline Mixed & 0 & $2(<1)$ & 0.15 & $23(1)$ & $17(1)$ & 0.74 \\
\hline Other & $4(<1)$ & $3(<1)$ & 0.75 & $27(1)$ & $28(2)$ & 0.10 \\
\hline Unknown & 0 & 0 & & $1212(52)$ & $830(52)$ & 0.33 \\
\hline \multicolumn{7}{|c|}{ Co-morbidities } \\
\hline Hypertension & $566(36)$ & $499(34)$ & 0.14 & $512(22)$ & $90(13)$ & 0.000 \\
\hline Diabetes Mellitus & $138(9)$ & $94(6)$ & 0.009 & $206(9)$ & $81(12)$ & 0.000 \\
\hline Lung Conditions & $92(6)$ & $91(6)$ & 0.76 & $59(3)$ & $88(13)$ & 0.000 \\
\hline Renal Impairment & $34(2)$ & $19(1)$ & 0.05 & $142(6)$ & $7(1)$ & 0.000 \\
\hline Liver Conditions & $36(2)$ & $24(2)$ & 0.17 & $29(1)$ & $8(1)$ & 0.01 \\
\hline $\mathrm{CVD}^{1}$ & $177(11)$ & $223(15)$ & 0.002 & $85(4)$ & $110(16)$ & 0.000 \\
\hline \multicolumn{7}{|c|}{ Performance status } \\
\hline 0 & $1357(87)$ & $1341(91)$ & 0.000 & $279(30)$ & $582(53)$ & 0.000 \\
\hline 1 & $190(12)$ & $128(9)$ & 0.001 & $452(48)$ & $391(36)$ & 0.000 \\
\hline 2 & $11(<1)$ & $7(<1)$ & 0.40 & 178 (19) & $103(9)$ & 0.23 \\
\hline 3 & $2(<1)$ & $1(<1)$ & 0.59 & $16(2)$ & $8(<1)$ & 0.49 \\
\hline 4 & 0 & 0 & & $16(2)$ & $6(<1)$ & 0.19 \\
\hline
\end{tabular}

${ }^{1}$ Cardiovascular disease.

\section{References}

1. Ozturk, C.N.; Kuruoglu, D.; Ozturk, C.; Rampazzo, A.; Gurunian Gurunluoglu, R. Plastic Surgery and the COVID-19 Pandemic: A Review of Clinical Guidelines. Ann. Plast. Surg. 2020, 85 (Suppl. S2), S155-S160. [CrossRef]

2. Kibbe, M.R. Surgery and COVID-19. JAMA 2020, 324, 1151-1152. [CrossRef] [PubMed]

3. WHO. WHO Director-General's Opening Remarks at the Media Briefing on COVID-19. 11 March 2020. Available online: https:/ / www.who.int/dg/speeches/detail/who-director-general-s-opening-remarks-at-the-media-briefing-on-covid19-11-march2020 (accessed on 28 January 2021).

4. $\quad$ Burden, E.G.; Walker, R.W.; Ferguson, D.J.; Goubran, A.; Howell, J.R.; John, J.B.; Khan, F.; McGrath, J.; Evans, J. The provision of a time-critical elective surgical service during the COVID-19 Crisis: A UK experience. Ann. R. Coll. Surg. Engl. 2021, 103, 173-179. [CrossRef]

5. Ji, C.; Singh, K.; Luther, A.Z.; Agrawal, A. Is Elective Cancer Surgery Safe During the COVID-19 Pandemic? World J. Surg. 2020, 44, 3207-3211. [CrossRef]

6. Lambertini, M.; Toss, A.; Passaro, A.; Criscitiello, C.; Cremolini, C.; Cardone, C.; Loupakis, F.; Viscardi, G.; Meattini, I.; Dieci, M.V. Cancer care during the spread of coronavirus disease 2019 (COVID-19) in Italy: Young oncologists' perspective. ESMO Open 2020, 5, e000759. [CrossRef]

7. Salute Md. Circolari del Ministero Della Salute. Available online: https://sites.google.com/uniurb.it/osscovid19/circolari/ circolari-del-ministero-della-salute (accessed on 12 February 2021).

8. Bresadola, V.; Biddau, C.; Puggioni, A.; Tel, A.; Robiony, M.; Hodgkinson, J.; Leo, C.A. General surgery and COVID-19: Review of practical recommendations in the first pandemic phase. Surg. Today 2020, 50, 1159-1167. [CrossRef]

9. Raymond, E.; Thieblemont, C.; Alran, S.; Faivre, S. Impact of the COVID-19 Outbreak on the Management of Patients with Cancer. Target Oncol. 2020, 15, 249-259. [CrossRef] 
10. Kane, A.D.; Paterson, J.; Pokhrel, S.; Berry, S.K.; Monkhouse, D.; Brand, J.W.; Ingram, M.; Danjoux, G.R. Peri-operative COVID-19 infection in urgent elective surgery during a pandemic surge period: A retrospective observational cohort study. Anaesthesia 2020, 75, 1596-1604. [CrossRef] [PubMed]

11. Hanna, T.P.; King, W.D.; Thibodeau, S.; Jalink, M.; Paulin, G.A.; Harvey-Jones, E.; O'Sullivan, D.E.; Booth, C.M.; Sullivan, R.; Aggarwal, A. Mortality due to cancer treatment delay: Systematic review and meta-analysis. BMJ 2020, 371, m4087. [CrossRef]

12. Maringe, C.; Spicer, J.; Morris, M.; Purushotham, A.; Nolte, E.; Sullivan, R.; Rachet, B.; Aggarwal, A. The impact of the COVID-19 pandemic on cancer deaths due to delays in diagnosis in England, UK: A national, population-based, modelling study. Lancet Oncol. 2020, 21, 1023-1034. [CrossRef]

13. Simoes, J.; Bhangu, A.; CovidSurg, C. Should we be re-starting elective surgery? Anaesthesia 2020, 75, 1563-1565. [CrossRef]

14. Sok, M.; Zavrl, M.; Greif, B.; Srpcic, M. Objective assessment of WHO/ECOG performance status. Support Care Cancer 2019, 27, 3793-3798. [CrossRef]

15. Daabiss, M. American Society of Anaesthesiologists physical status classification. Indian J. Anaesth. 2011, 55, 111-115. [CrossRef]

16. Dindo, D.; Demartines, N.; Clavien, P.A. Classification of surgical complications: A new proposal with evaluation in a cohort of 6336 patients and results of a survey. Ann. Surg. 2004, 240, 205-213. [CrossRef]

17. England PH. Coronavirus (COVID-19) Cases in London 2021. Available online: https:/ / coronavirus.data.gov.uk/details/cases? areaType=region\&areaName=London (accessed on 29 March 2021).

18. Salute Md. Covid-19—Situazione in Italia 2020. Available online: http://www.salute.gov.it/portale/nuovocoronavirus/ dettaglioContenutiNuovoCoronavirus.jsp?area=nuovoCoronavirus\&id=5351\&lingua=italiano\&menu=vuoto $($ accessed on 12 February 2021).

19. Italia PC. 2020 [cited 2021 February]. Available online: http:/ /www.protezionecivile.gov.it/ (accessed on 29 March 2021).

20. Givi, B.; Schiff, B.A.; Chinn, S.B.; Clayburgh, D.; Iyer, N.G.; Jalisi, S.; Moore, M.G.; Nathan, C.-A.; Orloff, L.A.; O’Neill, J.P.; et al. Safety Recommendations for Evaluation and Surgery of the Head and Neck During the COVID-19 Pandemic. JAMA Otolaryngol. Head Neck Surg. 2020, 146, 579-584. [CrossRef]

21. Ansarin, M. Surgical management of head and neck tumours during the SARS-CoV (COVID-19) pandemic. Acta Otorhinolaryngol. Ital. 2020, 40, 87-89. [CrossRef] [PubMed]

22. Maspero, M.; Mazzola, M.; Bertoglio, C.L.; Crippa, J.; Morini, L.; Magistro, C.; De Martini, P.; Gualtierotti, M.; Lombardi, P.M.; Ferrari, G. Major cancer surgery during the coronavirus pandemic: Experience from a tertiary referral center and COVID-19 hub in Northern Italy. Br. J. Surg. 2020, 107, e440-e441. [CrossRef] [PubMed]

23. Al-Quteimat, O.M.; Amer, A.M. The Impact of the COVID-19 Pandemic on Cancer Patients. Am. J. Clin. Oncol. 2020, 43, 452-455. [CrossRef]

24. Collins, P.M.; Madden, A.; O'Connell, C.; Omer, S.A.; Shakeel Inder, M.; Casey, R.G.; Flynn, R.J.; Thomas, A.Z.; Smyth, L.G.; Manecksha, R.P. Urological service provision during the COVID-19 period: The experience from an Irish tertiary centre. Ir. J. Med. Sci. 2020, 1-6. [CrossRef]

25. Maccagnano, C.; Rocchini, L.; Montanari, E.; Conti, G.N.; Petralia, G.; Dehò, F.; Bryan, K.-A.; Contieri, R.; Hurle, R. Overview of the italian experience in surgical management of bladder cancer during first month of COVID-19 pandemic. Arch. Ital. Urol. Androl. 2020, 92. [CrossRef]

26. Longo, F.; Trecca, E.M.C.; D’Ecclesia, A.; Copelli, C.; Tewfik, K.; Manfuso, A.; Pederneschi, N.; Mastromatteo, A.; Russo, M.A.; Pansini, A.; et al. Managing head and neck cancer patients during the COVID-19 pandemic: The experience of a tertiary referral center in southern Italy. Infect. Agent Cancer 2021, 16, 9. [CrossRef] [PubMed]

27. Smelt, J.; Santhirakumaran, G.; Vaughan, P.; Hunt, I.; Tan, C. Thoracic Surgery during Coronavirus Disease 2019 (COVID-19): The Experience of a Level 1 Trauma Center. Thorac. Cardiovasc. Surg. 2020, 1-7. [CrossRef]

28. Kamarajah, S.K.; Markar, S.R.; Singh, P.; Griffiths, E.A. Oesophagogastric Anastomosis Audit G. The influence of the SARS-CoV-2 pandemic on esophagogastric cancer services: An international survey of esophagogastric surgeons. Dis. Esophagus. 2020, 33, doaa054. [CrossRef] [PubMed]

29. Houvenaeghe, G.; Barrou, J.; Sabiani, L.; Blache, G.; Cohen, M.; Pignot, G.; Bannier, M.; Jauffret, C.; Rua, S.; Buttarelli, M.; et al. Surgical Oncologic Activity Before, During and after Pandemic COVID-19 Period in a French Cancer Center. J. Surg. Res. 2020, 3, 370-381. [CrossRef]

30. Apostolou, K.; Vogli, S.; Frountzas, M.; Syllaios, A.; Tolia, M.; Papanikolaou, I.S.; Schizas, D. Upper Gastrointestinal Cancer Management in the COVID-19 Era: Risk of Infection, Adapted Role of Endoscopy, and Potential Treatment Algorithm Alterations. J. Gastrointest. Cancer 2020, 1-7. [CrossRef]

31. Pavia, G.; Gargiulo, L.; Valenti, M.; Facheris, P.; Nucca, O.; Narcisi, A.; Borroni, R.G.; Costanzo, A. Skin cancers: How to balance the risks and benefits of surgery during COVID-19 pandemic (a Northern Italy single-center experience). Int. J. Dermatol. 2020, 59, 1287-1289. [CrossRef]

32. Bellato, V.; Konishi, T.; Pellino, G.; An, Y.; Piciocchi, A.; Sensi, B.; Siragusa, L.; Khanna, K.; Pirozzi, B.M.; Franceschilli, M.; et al. Screening policies, preventive measures and in-hospital infection of COVID-19 in global surgical practices. J. Glob. Health 2020, 10, 020507. [CrossRef]

33. Pelle, F.; Cappelli, S.; Graziano, F.; Piarulli, L.; Cavicchi, F.; Magagnano, D.; De Luca, A.; De Vita, R.; Pozzi, M.; Costantini, M.; et al. Breast cancer surgery during the Covid-19 pandemic: A monocentre experience from the Regina Elena National Cancer Institute of Rome. J. Exp. Clin. Cancer Res. 2020, 39, 171. [CrossRef] 
34. Evans, S.; Taylor, C.; Antoniou, A.; Aggarwal, T.; Burns, E.; Jenkins, J.T.; Miskovic, D. Implementation of a clinical pathway for the surgical treatment of colorectal cancer during the COVID-19 pandemic. Colorectal Dis. 2020, 22, 1002-1005. [CrossRef]

35. Sukumar, V.; Pandey, D.; Kumar, B.R.; Patel, S.; Pawar, T.; Rohila, J.; DeSouza, A.; Saklani, A. Colorectal Services in Covid-19 Times: Minimally Invasive Surgery and Enhanced Recovery, the Need of the Hour. Indian J. Surg. Oncol. 2020, 11, $297-301$. [CrossRef]

36. Perrone, A.M.; Dondi, G.; Giunchi, S.; De Crescenzo, E.; Boussedra, S.; Tesei, M.; D’Andrea, R.; De Leo, A.; Zamagni, C.; Morganti, A.G.; et al. COVID-19 free oncologic surgical hub: The experience of reallocation of a gynecologic oncology unit during pandemic outbreak. Gynecol. Oncol. 2020, 161, P89-P96. [CrossRef] [PubMed] 